\title{
Phase-Field Simulation for the Effects of Initial Vacancy Concentration and Annealing Temperature on Oxygen Clusters in Silicon Wafer
}

\author{
Xiao-Jun GUAN ${ }^{1,2, a}$, Xiang $\mathrm{JI}^{1,2, b}$ \\ ${ }^{1}$ School of Materials Science and Engineering, Shandong University, Jinan 250061, China; \\ ${ }^{2}$ State Key laboratory of Crystal Material, Shandong University, Jinan 250100, China \\ axjguan@sdu.edu.cn, bjx914234585@163.com
}

\begin{abstract}
Key words: phase field simulation; silicon wafer; low temperature annealing; initial vacancy concentration; annealing temperature; oxygen clusters.
\end{abstract}

\begin{abstract}
In order to investigate the dual effect of initial vacancy concentration and annealing temperature on oxygen clusters' evolution in RTP-treated silicon wafer during low temperature annealing, an established phase-field model and the optimum design method based on response surface was used to simulated the processes of oxygen clusters' evolution under their different combined conditions. The results show that the comprehensive influence of initial vacancy and annealing temperature on oxygen clusters mainly comes from their respective effects, and when initial vacancy concentration is higher than $1 \times 10^{15} \mathrm{~cm}^{-3}$ and annealing temperature changes from $1075 \mathrm{~K}$ to $1175 \mathrm{~K}$, the more numerous oxygen clusters can generate in the process of low temperature annealing.
\end{abstract}

\section{Introduction}

In recent years, the quality of $\mathrm{CZ}$ silicon enhances unceasingly with the development of IC manufacture and the improvement of annealing technology. American MEMC company has created "internal gettering" technics, that is, "rapid thermal processing (RTP)" method substitutes the first high temperature annealing of traditional "high-low-high" annealing technology and later two annealing remainss unchanged. The technique can control the formation and distribution of the oxygen precipitate by injecting numerous vacancies into silicon wafer to improve its quality and performance vastly ${ }^{[1]}$. In this technics, low temperature annealing becomes a link between RTP and final high temperature annealing. Therefore, the rational control of its parameters is a key to oxygen clusters' existing state, which is necessary to carry out the related simulation researches further.

So far, many scholars at home and abroad have done a lot of researches on the defects' behavior in annealed CZ silicon. R Falster and V. V. Voronkov ${ }^{[1,2]}$ investigated the behavior of point defects in silicon during annealing. Biao Wang ${ }^{[3]}$ and Li-ming $\mathrm{Fu}^{[4]}$ studied the effect of RTP on the formation mechanism of oxygen precipitate, respectively. But, the simulation on the evolution of oxygen clusters in RTP-treated silicon during low temperature annealing did not still carry out. We built a phase-field model which describe oxygen clusters' evolution in RTP-treated silicon during low temperature annealing and simulated the effects of annealing temperature on oxygen clusters' evolution $^{[5]}$. However, this research only found the influences of annealing temperature on oxygen clusters' evolution and did not consider the dual effect of it and initial vacancy concentration. Therefore, the processes of oxygen clusters' evolution under different combinations of initial vacancy concentration and annealing temperature are further simulated in this paper by using the model and the optimization design based on response surface.

\section{Simulation scheme and conditions}

In order to study the dual effect of both initial vacancy concentration and annealing temperature on oxygen clusters' evolution, considered their actual change scope and research need, the initial vacancy concentrations, $C_{v}$, of being $1 \times 10^{15} \mathrm{~cm}^{-3}, 1 \times 10^{14} \mathrm{~cm}^{-3}, 1 \times 10^{13} \mathrm{~cm}^{-3}, 1 \times 10^{12} \mathrm{~cm}^{-3}, 1 \times 10^{11} \mathrm{~cm}^{-3}$, 
and the annealing temperatures, T, of being $1225 \mathrm{~K}, 1175 \mathrm{~K}, 1125 \mathrm{~K}, 1075 \mathrm{~K}, 1025 \mathrm{~K}, 975 \mathrm{~K}, 925 \mathrm{~K}$, $875 \mathrm{~K}$, were chosen as the 40 different conditions for simulation, respectively.

According to the optimization design method ${ }^{[6]}, C_{\mathrm{v}}$ and $T$ were regarded as design variables $A$ and $B$, and the amount and area fraction of oxygen clusters were seleted as response values $R_{1}$ and $R_{2}$, respectively. And the simulation scheme was formulated by Design-Expert software ${ }^{[7]}$ and the simulation operations were finished by the established model and its applying program. The details information of the phase-field model, applying software, simulation area, and relevant simulation parameters in this paper are the same as that in Ref. [5].

Taking into account the probability characteristic of the model, the each response value in this paper is the average of five simulation results at the same simulation condition, and the related figures and analysis were given by Design-Expert software and Origin software, respectively.

\section{Simulation result analysis and discussion}

Fig. 1 represents the 3-D response surface of oxygen clusters' quantity, $R_{1}$. It is seen that higher initial vacancy concentration and annealing temperature correspond to the area more numerous oxygen clusters exist in. The fourth order regression model of this response surface is

$$
\begin{aligned}
& R_{1}=-2478.23-2384.56577 C_{\mathrm{v}}+41.71185 T-0.23653 C_{\mathrm{v}} T+268.3192 C_{\mathrm{v}}^{2}-0.060017 T^{2} \\
& +0.045536 C_{\mathrm{v}}^{2} T-2.22189 \times 10^{-4} C_{\mathrm{v}} T^{2}+1.02041 \times 10^{-5} C_{\mathrm{v}}^{2} T^{2}-14.30208 C_{\mathrm{v}}^{3}+3.97798 \times 10^{-5} T^{3} \\
& -1.76587 \times 10^{-3} C_{\mathrm{v}}^{3} T-3.63636 \times 10^{-8} C_{\mathrm{v}} T^{3}+0.29688 C_{\mathrm{v}}^{4}-9.48485 \times 10^{-9} T^{4} .
\end{aligned}
$$

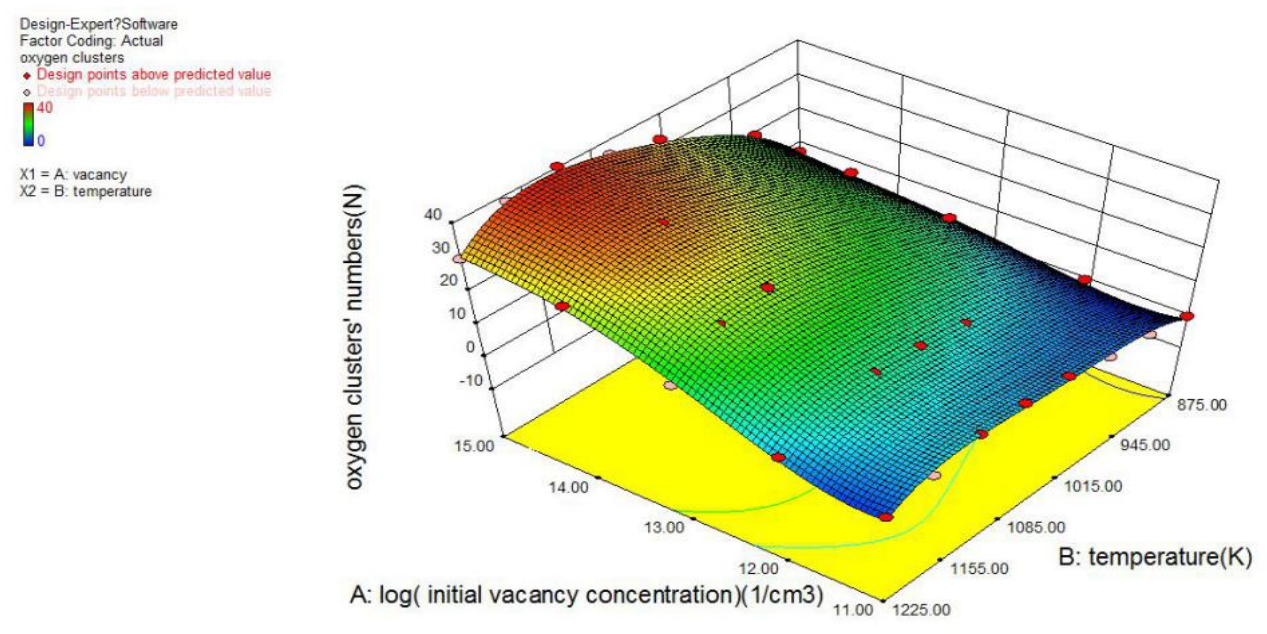

Fig. 1 Response surface of oxygen clusters' quantity, $R_{1}$, under different initial vacancy concentration and annealing temperature

It can be seen further in Fig. 2 that the oxygen clusters' amount increases with the increase of initial vacancy concentration, and the quantity first increases to the peak and then gradually reduces with raising annealing temperature. Obviously, the comprehensive influence laws of the two factors on the amount of oxygen cluster reflected by the response surface basically is in accordance with their individual effect laws.

Table 1 is the variance analysis results from the response surface regression model (Eq.1) and design variables' linear item, square one, cube one, quadruplicate one and the related cross items. In this table, $F$ represents the fluctuate degree of response target and its higher value means the greater influence of related variable on regression equation, and the greater are the complex correlation coefficient, $R$, and the regulation factor, $R_{\text {Adj }}$, the higher is the fitting degree of regression model ${ }^{[8]}$. It is shown from this table that the values corresponding to $F, R, R_{\text {Adj }}$ and the standard deviation, $S$, of 
this regression model are $158.03,0.9888,0.9826$, and 0.22 , respectively, which shows the higher fitting degree between the model itself and simulated data. For this model, the $F$ values of $A, B, B^{4}, A^{3}$, $A^{2}, B^{3}, A^{2} B$, and $A^{4}$ are greater and their values of (Prob>F) are less than 0.05 , which means the remarkable effects of these items on it. The strength order of these effects is $A>B>B^{4}>A^{3}>A^{2}>$ $B^{3}>A^{2} B>A^{4}$ and the former four items have more remarkable influence according to their $F$ values. And $A$ has the greatest effect, and $B, B^{4}$, and $A^{3}$ respectively follow it, and the effect of $A^{2} B$ is relatively little. The quantitative analysis mentioned above further confirms the conclusion resulted from Fig. 1 and Fig. 2, that is, the comprehensive influence of initial vacancy concentration and annealing temperature on oxygen clusters' quantity mainly depends on the superposition of their individual effects and is not matter with their interaction.

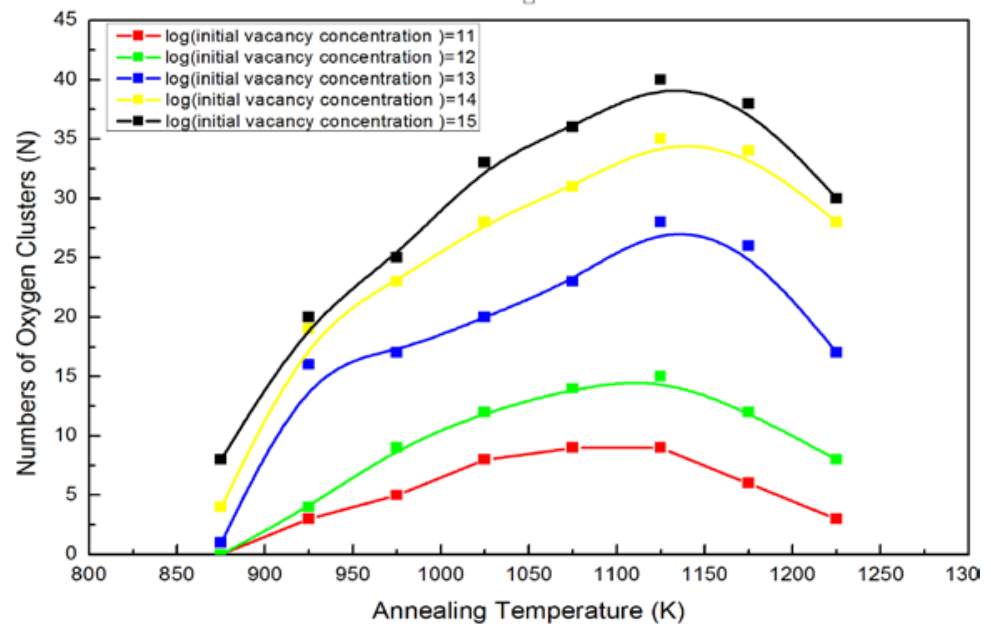

Fig. 2 The changes of oxygen clusters' amount under different conditional combination

Table 1 The variance analysis results of response surface regression model (ANOVA)

\begin{tabular}{cccccc}
\hline Source & Sum of Squares & DF & Mean Square & $F$ Value & Prob $>$ F \\
Model & 108.12 & 14 & 7.72 & 158.03 & $<0.0001$ \\
$A$ & 9.04 & 1 & 9.04 & 185.04 & $<0.0001$ \\
$B$ & 2.03 & 1 & 2.03 & 41.56 & $<0.0001$ \\
$A B$ & 0.025 & 1 & 0.022 & 0.44 & 0.5126 \\
$A^{2}$ & 0.39 & 1 & 0.39 & 8.06 & 0.0089 \\
$B^{2}$ & 0.018 & 1 & 0.018 & 0.37 & 0.5495 \\
$A^{2} B$ & 0.32 & 1 & 0.32 & 6.63 & 0.0163 \\
$A B^{2}$ & 0.14 & 1 & 0.14 & 2.90 & 0.1010 \\
$A^{2} B^{2}$ & 0.051 & 1 & 0.051 & 1.03 & 0.3189 \\
$A^{3}$ & 0.87 & 1 & 0.87 & 17.75 & 0.0003 \\
$B^{3}$ & 0.38 & 1 & 0.38 & 7.83 & 0.0097 \\
$A^{3} B$ & 0.015 & 1 & 0.015 & 0.31 & 0.5850 \\
\hline & & & & &
\end{tabular}




\begin{tabular}{|c|c|c|c|c|c|}
\hline$A B^{3}$ & $5.444 \times 10^{-3}$ & 1 & $5.444 \times 10^{-3}$ & 0.11 & 0.7413 \\
\hline$A^{4}$ & 0.21 & 1 & 0.21 & 4.20 & 0.0510 \\
\hline$B^{4}$ & 1.33 & 1 & 1.33 & 27.20 & $<0.0001$ \\
\hline \multicolumn{2}{|c|}{ Std. Dev. } & 0.22 & \multicolumn{2}{|c|}{$R$-Squared } & 0.9888 \\
\hline \multicolumn{2}{|c|}{ Mean } & 3.83 & \multicolumn{2}{|c|}{$R_{\text {Adj }}$-Squared } & 0.9826 \\
\hline \multicolumn{2}{|c|}{ C.V. \% } & 5.77 & \multicolumn{2}{|c|}{$R$ Pred -Squared } & 0.9659 \\
\hline \multicolumn{2}{|c|}{ PRESS } & 3.73 & \multicolumn{2}{|c|}{ Adeq Precision } & 47.291 \\
\hline
\end{tabular}

In order to reflect the accurate change of oxygen clusters' quantity in simulated area under the conditions of different initial vacancy concentrations and annealing temperatures, this paper further selects oxygen clusters area fraction, $R_{2}$, (that is the ratio of oxygen clusters' area and simulation one) as another response target, and the relevant simulation results are shown in Fig. 3.

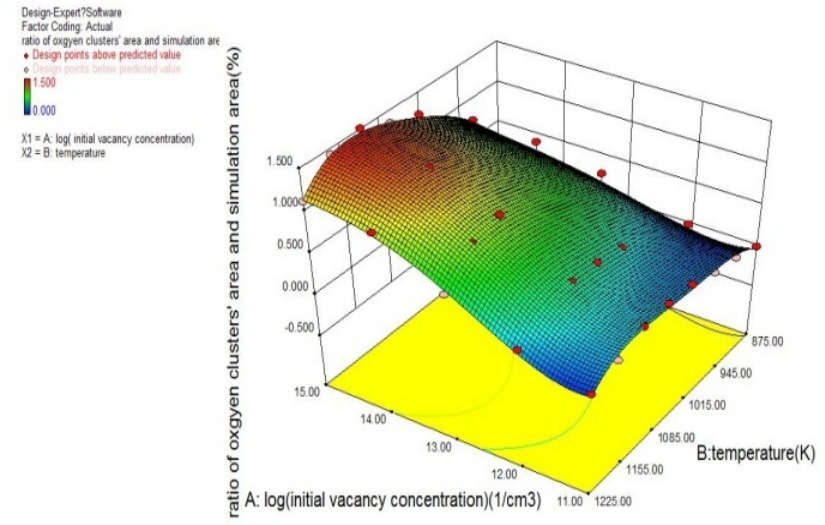

(a)

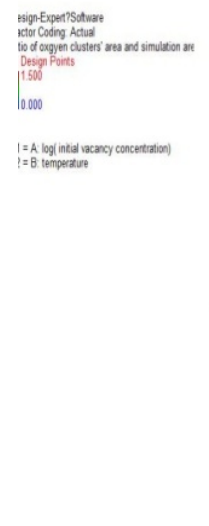

Fig. 3 Effect of initial vacancy concentration and annealing temperature on oxygen

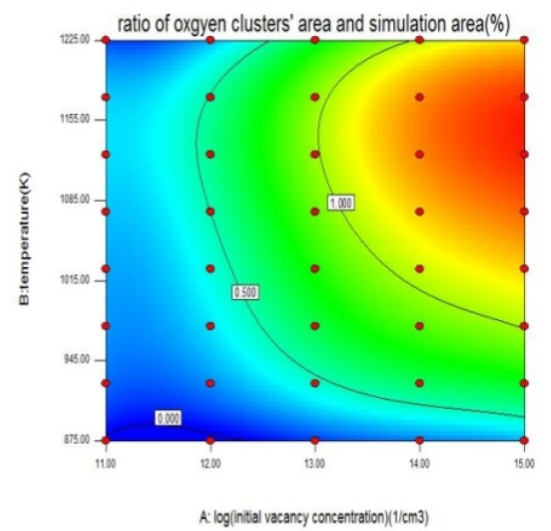

(D)

\footnotetext{
clusters area fraction, $R_{2}$
(a)response surface (b)equiv

(a)response surface (b)equivalence string diagram
}

The response surface shown in Fig. 3(a) is the similar as that in Fig. 1, which makes the oxygen clusters' size deviation to be ignored, so that either oxygen clusters' quantity or oxygen clusters' area fraction can be chosen as response target. Therefore, the principles of controlling initial vacancy concentration and annealing temperature gained from Fig. 3(b) and Fig. 2 are that when initial vacancy concentration reaches $1 \times 10^{15} \mathrm{~cm}^{-3}$ and annealing temperature varies within the range of 1075 $\mathrm{K} \sim 1175 \mathrm{~K}$, the most oxygen clusters could exist, and when initial vacancy concentration is less than $1 \times 10^{12} \mathrm{~cm}^{-3}$ and annealing temperature is below $900 \mathrm{~K}$, oxygen clusters could not form.

The above principles is the same as the experimental conclusions of "when annealing temperature is $923 \mathrm{~K}$ or $1073 \mathrm{~K}$ and initial vacancy concentration is higher than $1 \times 10^{12} \mathrm{~cm}^{-3}$, the core of oxygen precipitation can form effectively" mentioned in Ref. [9]. In addition, Ref. [1] pointed out that oxygen clusters can form massively when annealing temperature is $1073 \mathrm{~K}$, and Ref. [4] and Ref. [10] revealed that the optimum annealing temperature range for oxygen precipitation nucleation is $923 \mathrm{~K}$ $\sim 1173 \mathrm{~K}$ and the threshold of initial vacancy concentration is $5 \times 10^{11} \mathrm{~cm}^{-3} \sim 8 \times 10^{11} \mathrm{~cm}^{-3}$, which also proves that the simulation results in this paper are credible. 


\section{Conclusions}

Based on the response surface optimization design method and the phase field one, this paper finishes the simulation researches about the dual effects of initial vacancy concentration and annealing temperature on oxygen clusters in RTP-treated silicon wafer during low temperature annealing.

(1) The comprehensive influence of initial vacancy concentration and annealing temperature on o xygen clusters' quantity mainly depends on the superposition of their individual effects, namely, oxy gen clusters' quantity increases with the increase of initial vacancy concentration and first raises unti $\mathrm{l}$ the peak and then gradually reduces with increasing annealing temperature, and their interaction ha s little influence.

(2) During low temperature annealing, when initial vacancy concentration is higher than $1 \times 10^{15} \mathrm{~cm}^{-3}$ and annealing temperature varies in the ranges from $1075 \mathrm{~K}$ to $1175 \mathrm{~K}$, the amount of oxygen clusters is greater, and when initial vacancy concentration is less than $1 \times 10^{12} \mathrm{~cm}^{-3}$ and annealing temperature is lower than $900 \mathrm{~K}$, oxygen clusters could not exist.

\section{Acknowledgments}

This work was financially supported by the Opening Foundation Project from State Key Laboratory of Crystal Materials, Shandong University (grant no. KF1303).

\section{References}

[1] R. Falster, V. V. Voronkov: Materials Science and Engineering, Vol.73 (2000), p. 87-94.

[2] V. V. Voronkov, B. Dai, M. S. Kulkarmi: Comprehensive Semiconductor Science and Technology, No. 3 (2011), p. 81-169.

[3] Biao Wang: Effect of Rapid Thermal Progressing on Impurity Behavior in Czochralski Silicon (Zhejiang university master thesis, Hangzhou, 2011). In Chinese

[4] Li-ming Fu: Effects of Rapid Thermal Processing on Oxygen Precipitation and Internal Gettering in Czochralski Silicon (Zhejiang university doctoral thesis, Hangzhou, 2008). In Chinese

[5] Xiang Ji, Xiao-Jun Guan, Jin Wang: Advanced Materials Research, Vols. 1120-1121 (2015), p. 473-477.

[6] Xiang-hong Xu, Ming-zhu He: Experiment Design and Application of Design-Expert and SPSS (Science Press, Beijing, 2010). In Chinese

[7] Zhi-hong Zhang, Zhen He, Wei Guo: Journal of shenyang institute of aviation industry, Vol.24. No.1 (2007), p. 87-91. In Chinese

[8] Yan-hui Yang, Dong Liu, Zi-yan He: Rare metal materials and engineering, Vol.38. No.6 (2009), p. 1019-1024. In Chinese

[9] Seigo Kishino, Yoshiaki Matsushita, Masaru Kanamori, et al: Japanese Journal of Applied Physics, Vol.21. No.1 (1982), p. 516-525.

[10] H. Zimmermann and R. Falster: Appl. Phys. Lett., Vol. 60 (1992), p. 3250-3252. 